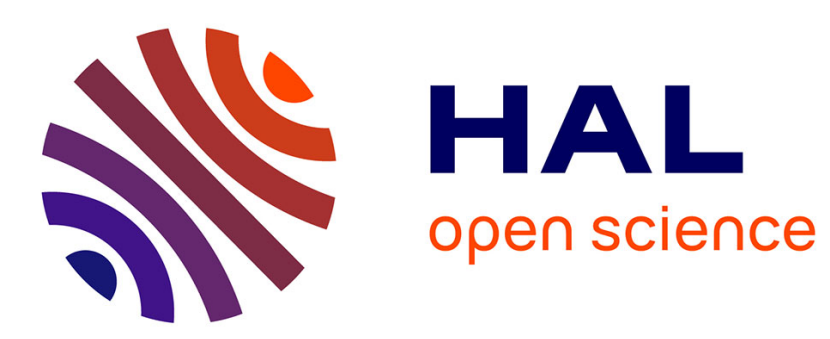

\title{
Prévision de la progression des fissures de fatigue dans les structures minces renforcées
}

\author{
J. Lemaitre
}

\section{To cite this version:}

J. Lemaitre. Prévision de la progression des fissures de fatigue dans les structures minces renforcées. Revue de Physique Appliquée, 1974, 9 (4), pp.667-672. 10.1051/rphysap:0197400904066700 . jpa00243833

\section{HAL Id: jpa-00243833 https://hal.science/jpa-00243833}

Submitted on 1 Jan 1974

HAL is a multi-disciplinary open access archive for the deposit and dissemination of scientific research documents, whether they are published or not. The documents may come from teaching and research institutions in France or abroad, or from public or private research centers.
L'archive ouverte pluridisciplinaire HAL, est destinée au dépôt et à la diffusion de documents scientifiques de niveau recherche, publiés ou non, émanant des établissements d'enseignement et de recherche français ou étrangers, des laboratoires publics ou privés. 


\title{
PRÉVISION DE LA PROGRESSION DES FISSURES DE FATIGUE DANS LES STRUCTURES MINCES RENFORCÉES (*)
}

\author{
J. LEMAITRE
}

Office National d'Etudes et de Recherches Aérospatiales (ONERA)

29, avenue de la Division-Leclerc, 92320 Châtillon, France

\begin{abstract}
Résumé. - Le comportement des structures fissurées sollicitées par des efforts dynamiques est fondé sur la connaissance du facteur d'intensité des contraintes qui détermine la vitesse de progression de la fissure par la loi de Paris-Forman, ou bien qui situe le danger de rupture fragile par comparaison avec sa valeur critique : la ténacité du matériau.

Un programme de calcul par éléments finis a été élaboré pour calculer le facteur d'intensité des contraintes en fonction de la longueur de fissure dans les structures planes raidies en mailles sollicitées en mode I. Le domaine de validité de la loi de Paris-Forman est étudié notamment sur le plan de l'histoire du chargement ; la loi ne peut être appliquée avec succès que si l'amplitude du chargement dynamique reste constante ou croît avec le temps.

Enfin, on décrit une méthode qui permet d'évaluer rapidement le facteur d'intensité des contraintes par mesures directes sur la structure fissurée chargée sans connaître les sollicitations au voisinage de la fissure.
\end{abstract}

Abstract. - The behaviour of cracked structures submitted to dynamic loading is based on the stress intensity factor concept associated to Paris-Forman law, to give the crack growth rate, or compared to the toughness of material to determine the conditions of brittle fracture.

A finite element program has been elaborated to compute the stress intensity factor as a function of crack length in plane substructures stiffened in mesh loaded in mode I. The validity of ParisForman law is studied particularly from the point of view of loading history. This law gives good results only if dynamic load amplitude is constant or increases in time.

A new method is described to determine quickly the stress intensity factor from direct measures on loaded cracked structure without any knowledge of loading in the crack vicinity.

1. Introduction. - Dans le domaine des cellules d'avions et plus généralement dans le domaine des structures souples performantes où l'apparition des fissures ne peut être évitée, il importe de savoir prévoir l'évolution de ces fissures sous l'action des charges dynamiques de service. Ce problème présente deux aspects.

1.1 Au niveau de la conception de la structure, cette prévision doit être effectuée par calculs «sur plans». La loi de base décrivant l'évolution d'une fissure dans un milieu bidimensionnel sollicité en chargement simple périodique est la loi de ParisForman [1]

$$
\frac{\mathrm{d} a}{\mathrm{~d} N}=f\left(\Delta K, \frac{K_{\mathrm{m}}}{K_{\mathrm{M}}}\right),
$$

où $a$ est la demi-longueur de la fissure,

$N$ est le nombre de cycles,

$K$ est le facteur d'intensité des contraintes dont la valeur minimale est $K_{\mathrm{m}}$, la valeur maximale $K_{\mathrm{M}}$;

$$
\Delta K=K_{\mathrm{M}}-K_{\mathrm{m}} \cdot
$$

$\left(^{*}\right)$ (Synthèse des travaux ONERA-SNIAS effectués dans le cadre du Contrat DGRST 71-72 885 « Mécanique de la rupture».)
Si le facteur $K$ est connu en fonction de la longueur de fissure $a$ et des charges extérieures, la loi de ParisForman peut s'écrire sous la forme d'une équation différentielle non linéaire dont la solution pour une condition initiale donnée $\left(N=N_{0} \Rightarrow a=a_{0}\right)$ fournit l'évolution cherchée de la fissure :

$$
a(N) \text {. }
$$

Pour obtenir la fonction $K(a)$ relative à des configurations de structures planes assez générales, on a élaboré un programme de calcul par éléments finis qui sera décrit sommairement au paragraphe 2 .

La plupart des structures réelles sont soumises à des chargements non périodiques, voire aléatoires. $\mathrm{Au}$ paragraphe 3 , on commentera des résultats expérimentaux qui permettent de cerner le domaine de validité de la loi de Paris-Forman.

1.2 Au niveau de la qualification d'un prototype ou de la maintenance des structures en service, lorsqu'une fissure est créé artificiellement (sur un prototype) ou détectée (sur une structure en service), il faut savoir évaluer le degré de danger qu'elle représente vis-à-vis de cas de chargements extérieurs donnés. La rupture fragile étant déterminée par la 
valeur critique du facteur d'intensité des contraintes, ou ténacité, $K_{\mathrm{c}}$ du matériau, le problème revient donc à évaluer la valeur du facteur $K$ correspondant à la configuration de structure étudiée pour la comparer à $K_{\mathrm{c}}$. Le plus souvent, on a besoin de ce renseignement rapidement, ce qui exclut une analyse théorique par calculs. On a développé une méthode résumée au paragraphe 4 qui permet d'évaluer le facteur d'intensité des contraintes à partir de mesures d'ouvertures de fissure réalisées «in situ » sur la structure chargée.

On se bornera ici à résumer les études effectuées sous les trois aspects : calcul du facteur d'intensité des contraintes par éléments finis, loi de progression de fissure, détermination du facteur $K$ par mesures in situ, chacun d'eux faisant l'objet d'une publication détaillée avec les références nécessaires qui ne seront pas rappelées ici.

2. Méthode de calcul par éléments finis du facteur d'intensité des contraintes. - La particularité essentielle du programme réalisé à l'ONERA [2] réside dans un découpage automatique qui permet de déterminer en un seul passage la fonction $K(a)$ pour autant de valeurs discrètes que l'on souhaite (une valeur représentant 3 minutes CPU sur IRIS 80). Il permet l'analyse des structures planes raidies en mailles c'est-à-dire les structures constituées de panneaux plans rectangulaires (plaques) et de raidisseurs rivés ou usinés (poutres). La fissure doit être normale ou parallèle aux raidisseurs, l'ensemble ayant deux axes de symétrie. La figure 1 montre quelques cas d'application issus de problèmes relatifs à des cellules d'avions.

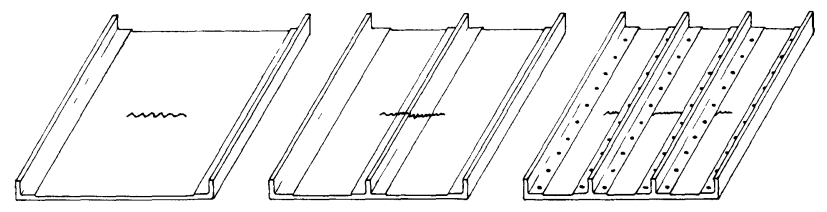

Fig. 1. - Exemples de sous-structures analysables.

Le chargement extérieur doit être un chargement plan et simple (toutes les forces croissent proportionnellement à un seul paramètre) de façon à assurer une croissance de la fissure dans son prolongement. Le matériau est élastique linéaire.

L'ensemble analysable constitue une sous-structure suffisamment importante pour qu'on puisse l'isoler du reste de la structure avec les conditions aux limites résultant du calcul général de la structure en l'absence de fissure.

Les calculs de contraintes et déformations sont effectués avec l'hypothèse des contraintes planes en élasticité linéaire. La zone plastique à la pointe de la fissure est négligée. La plaque fissurée est schématisée par des éléments triangulaires à 6 nœuds, conformes en déplacement. Pour obtenir une repré- sentation correcte du champ de contrainte théoriquement singulier à la pointe de la fissure, les éléments sont concentrés dans cétte zone, le découpage est réalisé à partir de points situés sur des cercles centrés à la pointe de la fissure. Les raidisseurs sont schématisés par des éléments de plaques pour les surépaisseurs et des éléments de poutres pour les âmes. Un exemple de découpage est donné sur la figure 2 .

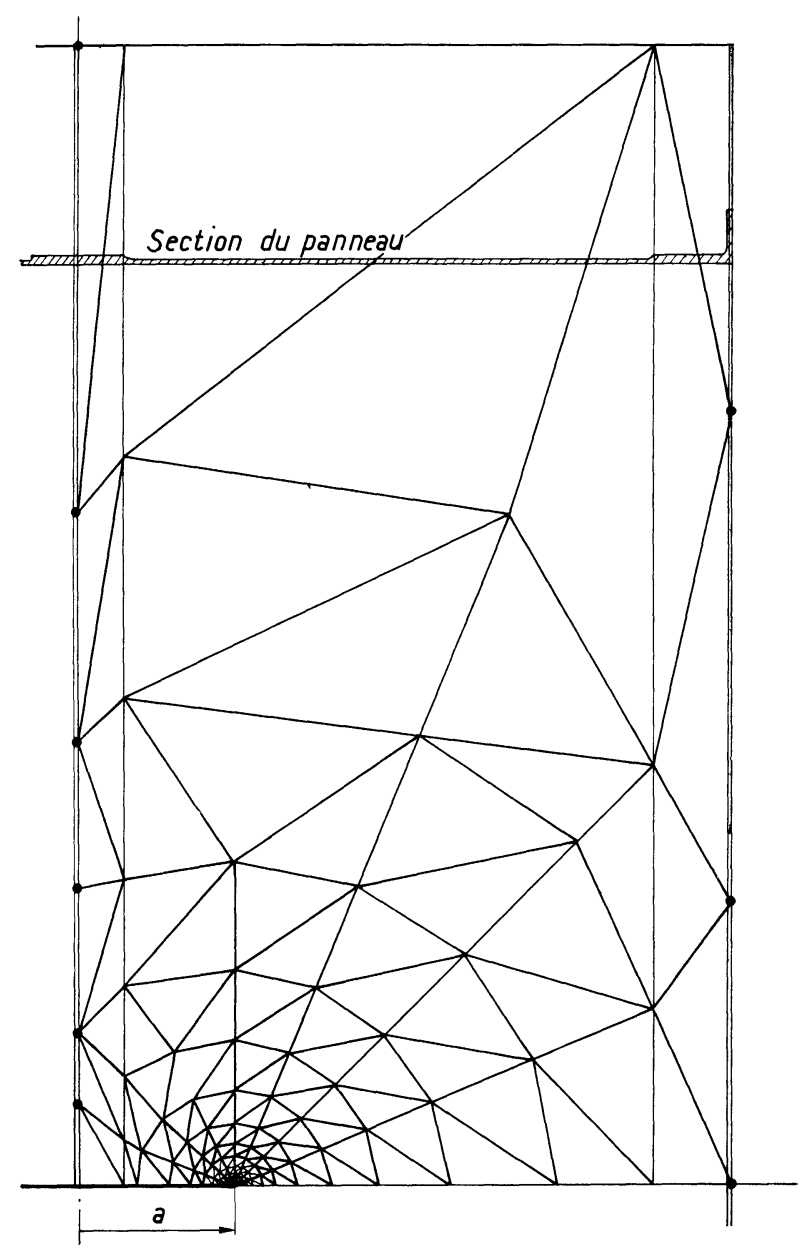

Fig. 2. - Exemple de découpage de $\frac{1}{4}$ de panneau.

Les charges extérieures sont représentées par des forces appliquées aux nœuds du contour extérieur.

Ce programme a été vérifié en calculant contraintes et déformations relatives à des configurations simples pour lesquelles on connaît les solutions analytiques et également en comparant les valeurs de déformations calculées à des mesures extensométriques par jauges effectuées sur des panneaux raidis fissurés.

Le facteur d'intensité des contraintes est calculé par la relation classique issu du bilan énergétique sur la structure élastique linéaire

$$
K=\left[\frac{E}{2} \frac{\mathrm{d} W}{\mathrm{~d} A}\right]^{1 / 2},
$$

où $E$ est le module d'Young. 
$\mathrm{d} W / \mathrm{d} A$ est la variation du travail des efforts extérieurs constants pour un accroissement d'aire de fissure $\mathrm{d} A$.

Cette relation qui permet de s'affranchir d'une représentation très fine du champ de contrainte à la pointe de la fissure est particulièrement bien adaptée au calcul par éléments finis. $W$ est calculé pour des valeurs discrètes de $A$ par :

$$
W\left(A_{j}\right)=\sum_{i} F_{i} \cdot v_{i}\left(A_{j}\right)
$$

$F_{i}$ et $v_{i}$ étant la force et le déplacement au nœud $i$. $\mathrm{d} W / \mathrm{d} A$ est obtenu par dérivation à partir d'un polynôme d'interpolation des $W\left(A_{j}\right)$.
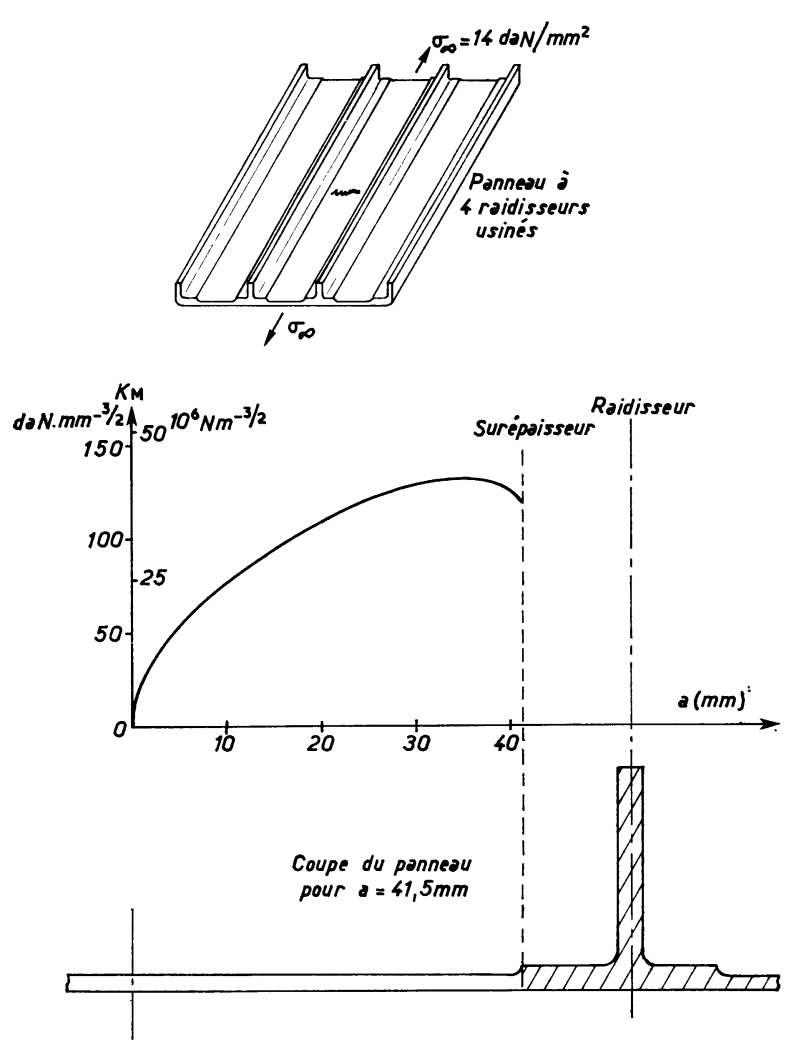

FIg. 3. - Evolution du facteur d'intensité des contraintes en fonction de la longueur de fissure.

A titre d'exemple, la figure 3 montre un résultat de calcul d'évolution du facteur d'intensité des contraintes $K_{\mathrm{M}}$ lorsque la fissure approche du raidisseur d'un panneau à 4 raidisseurs intégrés sollicité en mode I par une force uniformément répartie.

3. Domaine de validité de la loi de Paris-Forman. Une importante étude expérimentale a été conduite par l'Aérospatiale (Direction des études Toulouse) pour cerner le domaine d'application de la loi de Paris-Forman [3]

$$
\frac{\mathrm{d} a}{\mathrm{~d} N}=f\left(\Delta K, \frac{K_{\mathrm{m}}}{K_{\mathrm{M}}}\right)
$$

Trois alliages légers ont été étudiés à la température ambiante.

AU 2 GN T6 (alliage utilisé sur l'avion Concorde). AU 4 G1 T3/T4 (alliage utilisé sur l'avion Airbus). AZ 5 GU T73.

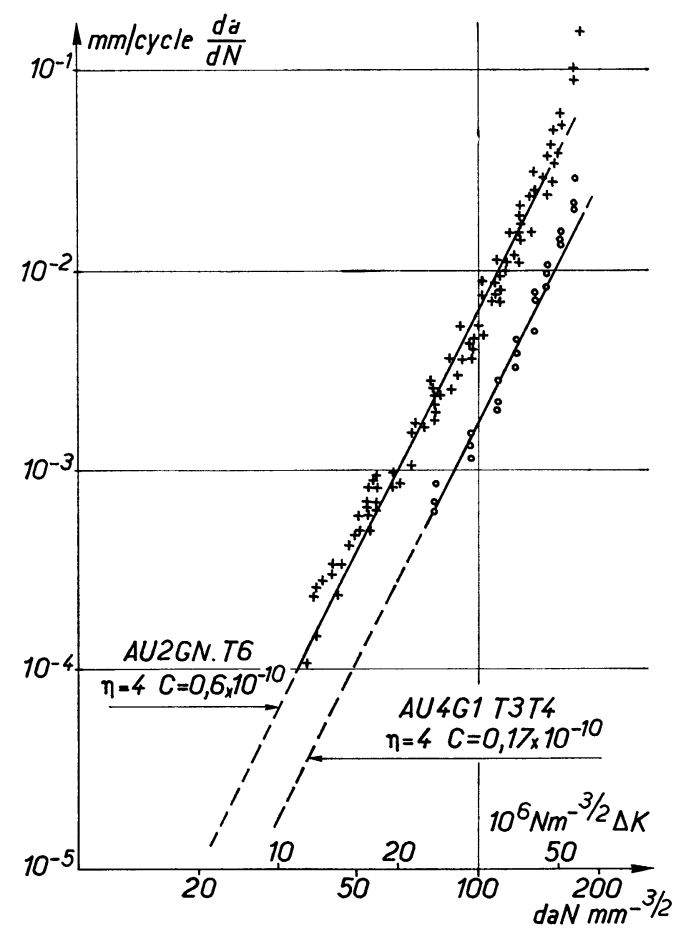

Fig. 4. - Loi de progression de fissure.

La figure 4 montre dans le plan $(\mathrm{d} a / \mathrm{d} N, \Delta K)$ des résultats d'essais de progression de fissures sur tôles nues soumises à un chargement périodique

$$
\sigma_{\infty}=7,5 \pm 6,5 \mathrm{~d} a N / \mathrm{mm}^{2},\left(\frac{K_{\mathrm{m}}}{K_{\mathrm{M}}}\right) \# 0 .
$$

3.1 Les limitations d'ordre géométrique sur la structure sont inhérentes à celles de la détermination du facteur d'intensité des contraintes. Pour les structures planes minces, le problème est l'influence de l'épaisseur. Le facteur d'intensité des contraintes étant calculé avec l'hypothèse des contraintes planes, les résultats d'essais sur tôles de différentes épaisseurs, traduits dans le plan $(\Delta K, \mathrm{~d} a / \mathrm{d} N)$ montrent que la fonction $f$ dépendrait de l'épaisseur, ce qui est bien sûr incompatible avec l'existence d'une loi intrinsèque au matériau. Cette influence est malgré tout assez faible, par exemple dans la gamme d'épaisseurs de 1 à $2 \mathrm{~mm}$, l'influence est masquée par la dispersion, par contre dans une gamme d'épaisseurs de 1 à $8 \mathrm{~mm}$, cette influence ne pourrait être négligée. Notons que l'épaisseur a une influence beaucoup plus nette sur la valeur critique de $K$ (pseudo-ténacité). Par exemple pour l'alliage AU 2 GN T6:

- épaisseur $1 \mathrm{~mm}$ :

$$
K_{\mathrm{c}}=170 \mathrm{~d} a N \mathrm{~mm}^{-3 / 2}\left(53,8 \times 10^{6} \mathrm{Nm}^{-3 / 2}\right)
$$


- épaisseur $2 \mathrm{~mm}$ :

$$
K_{\mathrm{c}}=195 \mathrm{~d} a N \mathrm{~mm}^{-3 / 2}\left(61,6 \times 10^{6} \mathrm{Nm}^{-3 / 2}\right) .
$$

3.2 Les limitations sur le cas de chargement sont classiques : chargement plan et simple (proportionnel) assurant une sollicitation de la fissure en mode I et sa croissance dans son prolongement.

3. 3 Ce sont les limitations relatives à l'histoire du chargement qui sont les plus contraignantes pour les applications pratiques. Pas ou peu d'études expérimentales ayant été faites en chargement alterné (traction-compression), il faut considérer que la loi de Paris ne peut être appliquée que dans les cas de chargements sollicitant la fissure en traction.

3.3.1 Influence de la sollicitation moyenne. - Dans la gamme des vitesses étudiées, les essais réalisés ont permis de mettre en évidence un paramètre global qui rend compte de l'effet de la sollicitation moyenne et qui conduit à une expression de la loi de Paris-Forman de la forme :

$$
\frac{\mathrm{d} a}{\mathrm{~d} N}=c\left[\frac{\Delta K}{\left[1-\frac{K_{\mathrm{m}}}{K_{\mathrm{M}}}\right]^{1 / 2}}\right]^{\eta},
$$

$c$ et $\eta$ étant des constantes caractéristiques du matériau.

3.3.2 Influence de la fréquence et de la forme des cycles. - Dans la gamme de fréquence de $10^{-2}$ à $50 \mathrm{~Hz}$, on peut considérer que l'effet de la fréquence n'est pas significatif. De même des résultats d'essais de progression de fissures sur panneaux plans sollicités sous charges périodiques en forme de créneaux avec deux temps de maintien en charge $\left(\Delta t_{1} / \Delta t_{2}=5\right)$ comparés avec les résultats d'essais sous sollicitation sinusoïdale de même amplitude montrent que les influences de la forme des cycles et du temps de maintien sont négligeables.

3.3.3 Cas des charges d'amplitude variable et aléatoire. - La loi de Paris-Forman reste valable tant que le facteur d'intensité des contraintes «crête» $K_{\mathrm{M}}$ est une fonction croissante du nombre de cycles. Dans le cas de chargements décroissants, on a vérifié que cette loi pouvait donner des résultats très erronés.

Ceci provient de l'effet de mémoire associé à la zone plastique à la pointe de la fissure. L'étude fine en est assez complexe mais on peut espérer que l'introduction d'un paramètre global supplémentaire (modèle de Wheeler par exemple [4]) dans la loi de Paris-Forman suffise pour prévoir la vitesse de progression de fissure dans le cas d'histoires complexes de chargement. Dans le cas de chargements aléatoires, si la bande d'amplitudes est assez étroite, la loi de Paris-Forman donne encore d'assez bons résultats mais pour les processus à large bande d'amplitude ou pour les cas de charges extrêmes à faible probabilité, il faut compliquer la loi de base.

Pour illustrer la prévision de la progression de fissure $a(N)$, on a porté sur la figure 5 le résultat de

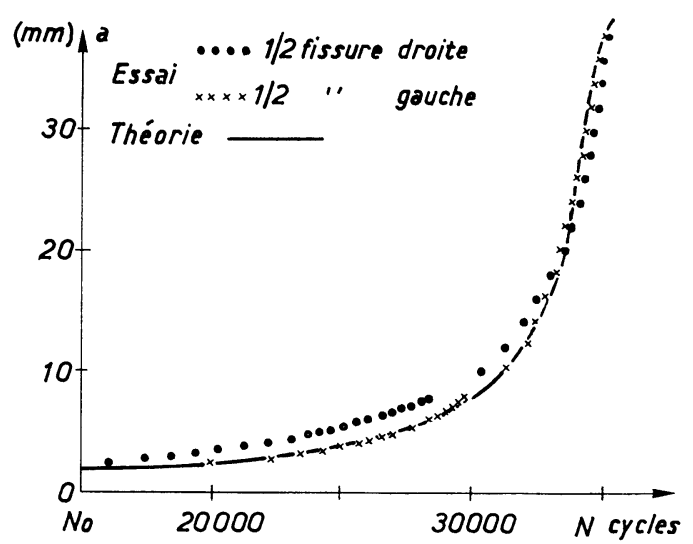

Fig. 5. - Prévision de la progression de fissure sur panneau à 4 raidisseurs usinés.

l'intégration de la loi de Paris-Forman avec la fonction $K(a)$ correspondant au panneau à 4 raidisseurs intégrés de la figure 3 :

$$
\begin{gathered}
\Delta K(a)=K_{\mathrm{M}}(a) \times \frac{\Delta F}{F_{\mathrm{M}}} \\
K_{\mathrm{m}} \# 0 \Rightarrow \frac{\mathrm{d} a}{\mathrm{~d} N}=c \Delta K^{\eta}
\end{gathered}
$$

avec la condition initiale $N=N_{0} \Rightarrow a=a_{0}$

$$
\Delta F=\text { Cte } \Rightarrow \int_{a_{0}}^{a} \frac{\mathrm{d} a}{K_{\mathrm{M}}(a)^{\eta}}=c\left[\frac{\Delta F}{F_{\mathrm{M}}}\right]^{\eta}\left[N-N_{0}\right] \text {. }
$$

La comparaison avec l'expérience correspondante montre que les différences entre la prévision et l'essai sont de l'ordre de grandeur des dispersions expérimentales, ce qui peut être considéré comme satisfaisant.

4. Evaluation du facteur d'intensité des contraintes par mesures directes sur la structure fissurée. - Soit une structure complexe (avion par exemple) formée dans la région fissurée de tôles minces planes ou à faible courbure renforcées par des raidisseurs disposés en mailles. La fissure coupe ou non un ou plusieurs raidisseurs, ses extrémités sont situées dans la tôle. Les efforts extérieurs constituent un chargement simple mais le champ des contraintes sur les mailles entourant la région fissurée est inconnu.

Pour évaluer par des mesures le facteur d'intensité des contraintes relatif à une configuration aussi complexe, on va se servir d'une équivalence avec un cas simple dont on connaît la solution analytique : le milieu bidimensionnel infini fissuré sollicité en mode I.

La méthode développée à l'ONERA [5] repose sur le principe suivant (Barrois 1969) : Pour toute structure fissurée, sollicitée en mode I et justiciable de l'hypothèse des contraintes planes en élasticité linéaire, il existe un domaine géométrique "d'équivalence » entourant la pointe de la fissure à l'intérieur duquel les champs de contrainte, déformation et déplacement 
s'identifient avec la solution de Muskhelishvili du milieu bidimensionnel infini fissuré.

Ce principe est cohérent avec l'existence du même type de singularité en $1 / \sqrt{r}$ du champ de contrainte élastique à la pointe de la fissure quelle que soit la structure considérée. Néanmoins, on a étudié la validité de ce principe en comparant les champs de déformation et de déplacement d'ouverture de la fissure de structures planes raidies déterminés soit par calculs (éléments finis) soit par expériences d'extensométrie, avec les champs correspondants des solutions de Muskhelishvili donnant le même facteur d'intensité des contraintes.

Pour déterminer le facteur d'intensité des contraintes $K$ d'une structure fissurée à l'aide de mesures « in situ ", on peut utiliser des mesures de déformations par jauges en amont de la pointe de la fissure ou bien des mesures d'ouverture de la fissure sous charge. C'est cette dernière technique qui a été développée car elle semble plus précise et surtout elle conduit à un temps d'exploitation beaucoup plus court.

Soit donc des mesures d'ouvertures de fissure $v_{i}$ correspondant aux abscisses $x_{i}$ dans le domaine d'équivalence et au chargement $\bar{F}$ sur la structure (Fig. 6).

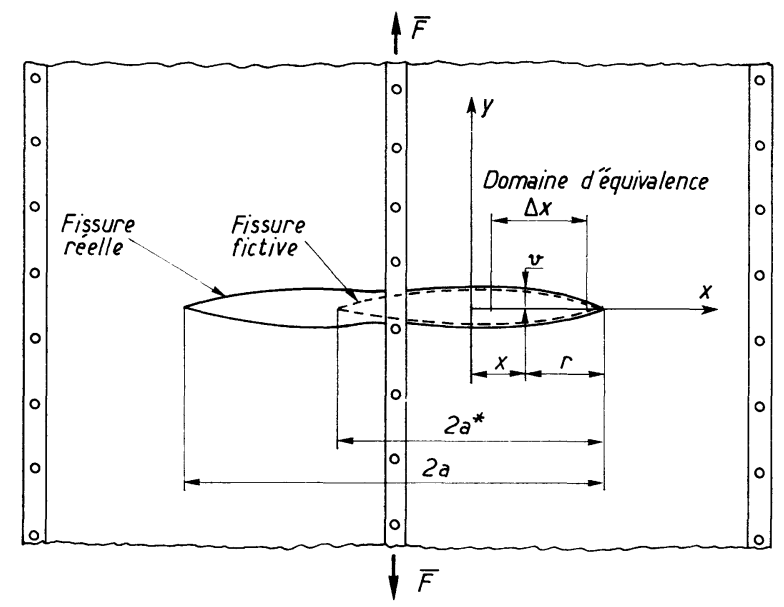

Fig. 6. - Définition de la fissure fictive équivalente sur la structure fissurée réelle.

On va identifier ce champ $v$ avec le champ donné par la solution de Muskhelishvili fonction de la contrainte infinie fictive $\sigma_{\infty}^{*}$ et de la longueur de fissure fictive $a^{*}$ qui constituent les deux inconnues $\mathrm{du}$ problème telles que d'après le principe de Barrois :

$K(\bar{F})$ structure $=K^{*}$ milieu bidimensionnel équivalent

$$
=\sigma_{\infty}^{*} \sqrt{\pi a^{*}} .
$$

L'équation du déplacement d'ouverture de Muskhelishvili est :

$$
v=\frac{2 \sigma_{\infty}^{*} a^{*}}{E}\left[1-\left[\frac{x}{a^{*}}\right]^{2}\right]^{1 / 2} .
$$

Le problème revient donc à déterminer $\sigma^{*}$ et $a^{*}$ qui satisfassent au mieux le système d'équations

$$
v_{i}=\frac{2 \sigma_{\infty}^{*} a^{*}}{E}\left[1-\left[\frac{x_{i}}{a^{*}}\right]^{2}\right]^{1 / 2}
$$

On peut utiliser la méthode des moindres carrés ou toute autre technique de lissage. Une technique commode et précise consiste à calculer $K$ par :

$$
K=\lim _{r \rightarrow 0}\left(v E \sqrt{\frac{\pi}{8 r}}\right) \text { avec } r=a^{*}-x
$$

car $v^{2} / r$ est linéaire en $r$.

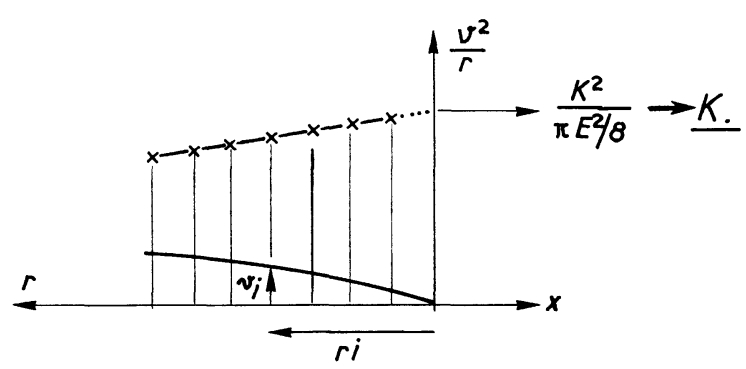

FIG. 7. - Technique de lissage par passage à la limite.

Pour réaliser ces mesures d'ouvertures, on a choisi une technique photographique qui consiste à faire des prises de vues de la fissure sur la structure chargée et à effectuer les mesures sur des agrandissements. On a réalisé à l'ONERA un appareil de macro-photo qui permet un grandissement de $100(13,18$ à la prise de vue sur polaroïd négatif $\times 7,58$ au tirage sur papier à stabilisation dont l'exploitation est quasi immédiate). Le champ objet est de $10 \mathrm{~mm}$, la précision à la lecture des ouvertures est de l'ordre de $\pm 2 \mu$.

D'un point de vue pratique, le domaine d'équivalences $x$ est défini par (Fig. 6) :

$$
\frac{a}{2}<\pi<\delta
$$

$\delta$ étant la longueur de la zone plastique.

Par ailleurs, pour éviter de prendre le risque de rupture de la structure, elle n'est chargée qu'à une fraction de $\bar{F}$, les valeurs $v(\bar{F})$ sont obtenues par extrapolation linéaire de valeurs obtenues pour des paliers de chargement inférieurs (par exemple $0,8 \bar{F}$, $0,6 \bar{F}, 0,4 \bar{F}, 0,2 \bar{F})$.

Enfin, la technique des moindres carrés et la technique de la limite permettent d'éliminer l'incertitude sur la mesure des abscisses engendrée par la mauvaise définition pratique de la pointe de la fissure. 
La méthode a été appliquée à un certain nombre de cas jugés difficiles et significatifs pour une vérification: des panneaux raidis avec des fissures près des raidisseurs ou sous des raidisseurs rivés. Le tableau de la figure 8 montre l'ensemble des résultats comparés à des valeurs de $K$ calculées par éléments finis et aux valeurs calculées par la loi de fissuration pour la valeur de vitesse $\mathrm{d} a / \mathrm{d} N$ mesurée expérimentalement. Sauf pour les valeurs de $K$ proches de $K_{\text {c }}$ (qui correspondent à des zones plastiques très importantes), on peut là encore considérer que la classe de précision de la méthode est du même ordre que la dispersion inhérente aux matériaux: $\pm 5 \%$.

A la demande des services officiels, cette méthode a été utilisée en novembre et décembre 1973 au Centre d'Essais Aéronautiques de Toulouse pour aider à la réalisation d'expériences de fissuration sur l'avion prototype "Corvette» dans le cadre des essais de certification.

\begin{tabular}{|c|c|c|c|c|}
\hline & $\begin{array}{r}\text { VALEURS DE } \\
\text { METHODE PAR } \\
\text { MESURES D'OUVEF }\end{array}$ & TURES & & \\
\hline $\begin{array}{l}\text { STRUCTURE } \\
\sigma_{\infty}=14 \mathrm{doN} / \mathrm{mm}^{2}\end{array}$ & $\begin{array}{c}\text { TECHNIQUE } \\
\text { DES } \\
\text { MOINDRES CARRES }\end{array}$ & $\begin{array}{l}\text { TECHNIQUE } \\
\text { DE LA } \\
\text { LIMITE }\end{array}$ & $\begin{array}{l}\text { VALEURS DE K } \\
\text { CALCULEES PAR } \\
\text { ELEMENTS FINIS }\end{array}$ & $\begin{array}{l}\text { VALEURS DE K } \\
\text { DETERMINES } \\
\text { PAR LA LOI } \\
\frac{d a}{d N}=f(\Delta K)\end{array}$ \\
\hline$\stackrel{20}{a}=50 \mathrm{~mm}$ & 133 & 132 & 130 & 127 \\
\hline$q^{2 a=102}$ & 216 & 222 & 193 & 191 \\
\hline$\Omega$ & 173 & 176 & 175 & 185 \\
\hline$[\Omega]^{20}=80,3$ & 137 & 136 & 131 & 132 \\
\hline ( $]^{2 a}=102$ & 192 & 195 & 180 & 190 \\
\hline ת & 159 & 159 & 157,5 & 163 \\
\hline
\end{tabular}

Fig. 8. - Résultats d'application de la méthode de détermination de $K$ par mesures " in situ »

$\left(K\left(N \mathrm{~m}^{-3 / 2}\right)=0,3162 \times 10^{6} \mathrm{~K}\left(\mathrm{~d} a N \mathrm{~mm}^{-3 / 2}\right)\right)$.

\section{Bibliographie}

[1] IRwIN, G. R., PC Paris, Fundamental aspects of crack growth and fracture (Liebowitz Academic Press) 1971, Chapitre 1 du Volume 3 de « Fracture ».

[2] Chaboche, J. L., Monthulet, A., Calcul du facteur d'intensité des contraintes pour la prévision de la progression des fissures. Publication ONERA à paraître (1974).

[3] LOUBET, R., Etude de propagation de fissures sur alliages légers.
Rapport d'étude $\mathrm{n}^{\circ} 437$ 544/73. Aérospatiale. Direction Etudes Toulouse (18. 9. 1973).

[4] WheELER, O. E., Spectrum loading and crack growth. ASME paper no 71-metx (1972).

[5] Lemaître, J., Monthulet, A., Recourcé, J. C., Evaluation du facteur d'intensité des contraintes par mesures directes sur la structure fissurée. Publication ONERA à paraître (1974). 